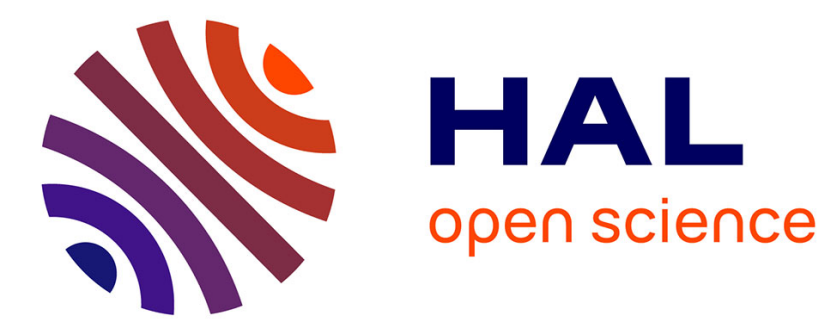

\title{
Les fonctions du tourisme obscur
}

\author{
Nathanaël Wadbled
}

\section{To cite this version:}

Nathanaël Wadbled. Les fonctions du tourisme obscur. Teoros. Revue de recherche en tourisme, 2016,

Tourisme noir ou sombre tourisme?, 35 (1), pp.[En ligne]. hal-01657762

\section{HAL Id: hal-01657762 \\ https://hal.science/hal-01657762}

Submitted on 7 Dec 2017

HAL is a multi-disciplinary open access archive for the deposit and dissemination of scientific research documents, whether they are published or not. The documents may come from teaching and research institutions in France or abroad, or from public or private research centers.
L'archive ouverte pluridisciplinaire HAL, est destinée au dépôt et à la diffusion de documents scientifiques de niveau recherche, publiés ou non, émanant des établissements d'enseignement et de recherche français ou étrangers, des laboratoires publics ou privés. 


\title{
Téoros
}

Revue de recherche en tourisme

35, 1 | 2016 :

Tourisme noir ou sombre tourisme?

Tourisme noir ou sombre tourisme?

\section{Les fonctions du tourisme obscur}

\section{Canevas d'une revue de littérature sur l'expérience de visite des lieux de génocide}

\author{
NATHANAËL WADBLED
}

\begin{abstract}
Au-delà des études de cas, de nombreuses études générales sur le tourisme obscur s'attachent à définir sa fonction spécifique sociale et culturelle. Indépendamment des caractéristiques propres de chaque site visité, ou de chaque touriste, est alors défini un certain mode de relation au patrimoine. Il se distingue du tourisme culturel historique au sens où il n'est pas intéressé par la transmission d'un savoir historien contextualisé, mais par l'éducation à des valeurs morales ayant une dimension civique. Cette situation est l'occasion d'un certain nombre de critiques. Au niveau du contenu transmis, la mise en avant de la transmission de valeurs aurait comme effet une déhistoricisation des événements. Au niveau de la manière dont il est transmis, le tourisme obscur risquerait de devenir une pratique récréative, car cette éducation s'adresse au ressenti des visiteurs et non à leur intelligence réflexive.
\end{abstract}

\section{Index terms}

Mots-clés : devoir de mémoire, éducation, identité, patrimoine, trivialisation.

\section{Full text}




\section{Un point de vue sur les musées et les sites historiques}

Dans la mesure où l'expression tourisme obscur (dark tourism) a dépassé l'usage particulier proposé par Malcom Foley et John Lennon en 1996, elle s'est imposée pour désigner généralement toutes visites de lieux associés à la mort et à la souffrance et en particulier les lieux de massacres de masse et de génocides contemporains. Pris en ce sens général, cette pratique touristique est étudiée selon trois perspectives qui sont des choix à la fois épistémiques et méthodologiques. Il s'agit soit d'analyses générales sur la fonction sociale et culturelle de ce tourisme indépendamment de la spécificité des différents sites, soit d'études de lieux particuliers qui décrivent leur agencement et insistent sur les particularités de chaque site, soit d'enquêtes sur les motivations des visiteurs. Ces trois approches correspondent à trois contextes que les muséologues John Falk et Lynn Dierking (2000) reconnaissent comme définissant les conditions de l'expérience de visite des touristes : social, physique et personnel.

S'il est possible d'identifier les fonctions culturelles comme un élément du contexte social, c'est au sens où le sociologue Maurice Halbwachs (1997) observe que le fait de percevoir d'une certaine manière signifie se placer comme membre d'un certain groupe social. Lorsqu'un individu perçoit quelque chose, il se positionne d'une certaine manière dans un groupe virtuel qu'il reconnaît et dans lequel il se reconnaît à ce moment-là. Il adopte alors le point de vue de cette position, c'est-à-dire d'un groupe social donné : en réalité, il n'est jamais seul puisqu'il actualise une manière de percevoir et de comprendre les choses qui est celle d'un groupe virtuel. C'est en ce sens que cette approche peut être dite fonctionnaliste (Malinowski, 1970 ; Deleuze, 1989). Les caractéristiques de cette position définissent le point de vue d'un groupe social auquel l'individu appartient au moment où il s'y place. Il s'agit de rendre compte de l'effet utile de cette position, c'est-à-dire des fonctionnements et des conditions de fonctionnement d'un dispositif social où une tâche ou une conduite serait imposée aux individus s'y plaçant. Le fonctionnement est considéré comme étant premier par rapport à ce qui fonctionne en tant que chose, de sorte que les caractéristiques propres du vécu subjectif des sites visités et des visiteurs seraient indifférentes. Cela permet de spécifier une catégorie d'expérience, ou ce que Halbwachs nomme un courant de mémoire, qui constitue les conditions transcendantales de toute expérience particulière. Cet intérêt pour la fonction sociale est développé dans de nombreuses recherches sur le tourisme. C'est en particulier celle suivie par John Lennon et Malcom Foley dans l'ouvrage qui a popularisé le terme de tourisme obscur. Pour cette raison, cette expression peut parfois désigner ce cadre méthodologique, au lieu de prendre un sens descriptif désignant tout ce qui renvoie au tourisme associé à la mort et à la souffrance. Ces travaux cherchent généralement à caractériser le phénomène d'une manière générale. L'enjeu est de déterminer à quel besoin répond la visite et dans quelle mesure elle répond à une demande ou à une exigence sociale. L'intérêt se porte alors sur l'offre touristique et sur la signification de la visite en tant que pratique collective effectuée par les membres d'une communauté donnée. Les sites associés au tourisme obscur sont alors définis comme engageant une expérience sociale particulière. Ils ne sont pas simplement une version sombre des musées d'histoire qui ne s'en distingueraient que sur le plan de la morbidité des événements évoqués.

3 La définition du tourisme obscur se fait indépendamment des lieux spécifiques et des motivations particulières de leurs visiteurs. Les études de cas particuliers valent comme exemples illustrant un type de pratique défini par son utilité dans l'organisation sociale 
générale. Les contextes physiques et personnels qui caractérisent particulièrement et spécifiquement chaque expérience en tant que situation particulière sont subsumés sous sa fonction qui définit la manière dont les individus vont l'envisager. L'objet, que ce soit le lieu visité ou le visiteur, serait alors relativement indifférent à la réalisation de la fonction. S'inscrire dans cette approche ne signifie donc pas nécessairement que tous les lieux et tous les visiteurs sont identiques, mais que ce ne sont pas leurs spécificités qui peuvent permettre de comprendre ce que les lieux montrent ou ce que leurs visiteurs vivent. Si les travaux sur le tourisme obscur ne thématisent généralement pas explicitement la question de l'expérience spécifique des visiteurs, il est possible d'en déduire les caractéristiques en termes de fonction sociale et culturelle.

La plupart des travaux qui s'attachent à définir les caractéristiques générales du tourisme obscur en tant que pratique sociale peuvent être considérés comme s'inscrivant dans cette perspective fonctionnaliste. Si Lennon et Foley définissent en particulier le contenu du tourisme obscur comme étant un certain rapport aux événements qui « posent question ou introduisent anxiété et doute au sujet de la modernité et de ses conséquences » $(2000: 12)^{1}$, cette définition demande cependant à être précisée. Le tourisme obscur apparaît dans ces travaux comme ayant une certaine relation aux musées et aux sites historiques, visant ce qui en eux donne et transmet des valeurs morales ayant généralement une forte dimension identitaire. Il s'agit donc d'éduquer à des valeurs, par opposition au tourisme culturel historique intéressé par des informations historiques destinées à être apprises ${ }^{2}$. La relation ainsi instaurée est réputée se fonder sur une attention et une réceptivité centrées sur le ressenti des visiteurs, plus que sur une concentration réflexive permettant d'apprendre ou d'intégrer un savoir théorique ou historien. Le tourisme obscur se distingue ainsi d'un intérêt pour l'apprentissage d'informations historiques aux niveaux à la fois du contenu et de la manière dont cette transmission se fait. Si cette façon de caractériser le tourisme obscur a souvent la forme d'une critique considérant cette pratique comme inférieure à celle du tourisme culturel, Philip Stone affirme explicitement qu'il faut prendre en compte la fonction productrice d'expériences du tourisme obscur et non simplement en faire le symptôme d'une perte du sens (2009: 71).

\section{Une éducation morale négative}

Le tourisme obscur est généralement présenté comme étant avant tout une réflexion critique permettant aux visiteurs de définir leur identité historique et morale. C'est en tant que telle que la commémoration ne sous-entend pas la perte des parties sombres ou douloureuses de l'histoire : ces événements ne sont pas nécessairement déniés ou minimisés dans leur irréductible dissonance par la dimension identitaire de leur expérience patrimoniale. La commémoration les inscrit dans une pratique patrimoniale de l'histoire au sens défini par le géographe David Löwenthal (1998 : 127-145) : son enjeu n'est pas de conserver, mais d'utiliser ou de "consommer » un événement, en apportant aux visiteurs la possibilité de spécifier leur identité historique et sociale. Chacun tire de sa visite une conscience à la fois de sa propre identité historique et des préceptes permettant de juger l'action présente.

$6 \quad$ Le présent appartient à la même histoire continue et les individus du passé ne sont donc pas considérés comme des ancêtres, mais comme des hommes similaires à ceux présents. En même temps, cette continuité permet de tirer des leçons du passé susceptibles de servir. Cette démarche s'inscrit dans une certaine conception de l'histoire héritée de sa pratique pré-moderne : l'historia magistrae vita, selon laquelle 
les situations passées servent de modèle au présent (Hartog, 2003 : 38-50). Contrairement à l'histoire qui établit une coupure épistémique entre le présent et le passé et définit chaque situation comme unique (de Certeau, $1975: 16$ ), le tourisme patrimonial de musées ou de sites historiques a ainsi une dimension pédagogique distincte de la fonction d'apprentissage de l'histoire cherchant à connaître le passé en lui-même. En même temps, il y a une spécificité du tourisme obscur à l'intérieur du tourisme patrimonial : la relation patrimoniale se fait de manière négative. Les événements sont visés en tant que participant de ce que Tony Seaton qualifie d'autréité (othering) (2009 : 78). Ces événements sont perçus comme des repoussoirs. Une identité historique et des valeurs morales sont définies en opposition.

\section{Définir une identité historique}

Paul Williams insiste emblématiquement sur ces deux dimensions et en explicite les enjeux d'une manière particulièrement intéressante dans la mesure où il fait jouer ensemble des éléments ou des fonctions du tourisme obscur pouvant apparaitre séparés dans différentes analyses. Avec un certain nombre d'exemples, il montre que les événements visés par le tourisme obscur sont exclus du récit linéaire et normal de l'histoire (2007 : 122, 138, 158, 165). Ils introduisent des ruptures et apparaissent comme obéissant à d'autres logiques que celles de cette histoire qui est encore celle des visiteurs. La conséquence est que ces événements sont incompréhensibles : ils échappent à la rationalité historique permettant à la fois d'en rendre compte et de les inclure dans le récit de ce qui est reconnu par les visiteurs comme étant leur histoire. Cependant, dans le même temps, visiter des espaces associés à ces événements les inscrit dans cette histoire. Un musée-mémorial intègre un événement dans l'histoire : il établit des relations intimes avec l'événement tout en le mettant à distance. Il y a à la fois une rupture vis-à-vis des conditions de l'événement et une continuité : l'événement est en un sens compris comme étant incompréhensible (Wadbled et Farache, 2013 : 132-134).

La manière dont John Lennon et Malcolm Foley en parlent montre bien une telle situation en apparence paradoxale. Ils affirment globalement que le tourisme obscur instaure un doute sur l'histoire dans laquelle les visiteurs se reconnaissent (Lennon et Foley, 2000 : 22, Phillips, 2004 : 95-97). L'événement mis à distance apparaît alors non comme extérieur, mais menaçant de l'intérieur : les événements perçus dans la perspective du tourisme obscur sont un danger qui guette et menace cette histoire. Ils y restent présents, mais comme quelque chose qui ne devrait pas exister. Si Lennon et Foley (2000 : 31) affirment à quelques reprises que de tels événements sont regardés comme une extériorité radicale rendant inadéquate toute explication et compréhension, il faut sans doute prendre ces formulations pour des excès de langage. Sans que cela implique leur exclusion de l'histoire, ces événements apparaissent bien comme ne faisant pas sens dans le récit historique de la même manière, par exemple, que les batailles articulées à l'héroïsme ou à la défense de la patrie. C'est ainsi que la commémoration ne suppose pas la perte des parties sombres ou douloureuses de l'histoire.

9 C'est d'ailleurs dans la mesure où il faut en même temps les tenir à distance et les reconnaître comme possibles que cette construction identitaire est indissociable d'une condamnation morale. S'il est possible de mettre en avant la seule dimension morale (Stone $2009:$ 70), Williams propose une vision plus complexe où le tourisme obscur apparaît comme étant une leçon indistinctement historique et morale (2007: 131, 149, 
162). Dans cette perspective sont définies des valeurs en fonction desquelles ce qui pourrait entraîner le retour de tels événements est jugé négativement et où ce qui garantit qu'ils ne se reproduiront pas est jugé positivement. Avec des valeurs de tolérance, de liberté, de paix et de démocratie, il s'agit à la fois - négativement d'éviter que des tragédies comparables se reproduisent et - positivement - de réparer ce qui a eu lieu en reprenant le fil et la logique humaniste d'une histoire interrompue. Dans la première perspective s'inscrit la dénonciation des horreurs qui sont advenues et dans la seconde, l'attention à la souffrance des victimes. C'est à ce double niveau que le tourisme obscur s'inscrit dans la pratique plus générale de ce qui est appelé en France le devoir de mémoire caractérisé par cette double attitude (Ledoux, 2012). Elles passent toutes deux par une culpabilisation de ne pas connaitre cette histoire et de ne pas se rendre compte des horreurs vécues par les victimes.

Cette façon de fonder des valeurs de manière négative sur le passé est largement et doublement critiquée. La critique est construite soit en mettant l'accent sur le rapport au passé qui empêcherait de vivre le présent ou de se projeter vers le futur, soit en insistant sur la négativité remplaçant les modèles positifs. La première critique est celle par exemple d'Andreas Huyssen qui affirme que le tourisme obscur a une fonction de compensation face au présent qui est la marque d'une situation éthique et politique ne parvenant pas à trouver en elle-même ses ressources pour distinguer le bien du mal (Huyssen, 2000 ; Misztal, 2004). Le passé, loin d'être une source d'inspiration pour le présent, serait ce qui l'aliène dans une peur de l'oubli. À côté de cette critique, Peter Novick (1999) adhère par exemple à la nécessité de considérer le présent comme une réponse au passé, tout en jugeant qu'il faudrait le faire de manière positive par une identification aux héros et non par une commémoration des victimes (voir aussi Dean, 2003). Il y aurait donc une culture de la victime ou de la victimisation fondant les valeurs sur l'indignation au lieu de le faire sur l'admiration. L'éducation morale n'est généralement pas mentionnée de manière positive.

\section{Le sentiment d'appartenir à une communauté universelle}

Contrairement à un tourisme historique pouvant être qualifié de positif, la dénonciation n'est pas compensée par l'affirmation de l'héroïsme ou de la gloire qu'il faudrait continuer, mais est renforcée par celle de la vulnérabilité qu'il faut protéger au contraire de ce qui a été fait. L’enjeu est donc de faire prendre conscience de la nécessité de promouvoir certaines valeurs afin d'encourager certains comportements. Si les valeurs et l'identité historique qu'elles portent sont en elles-mêmes universelles, à l'échelle locale, la dimension morale peut prendre une signification politique et civique nationale sous la forme d'une vigilance citoyenne envers ce qui est identifié comme conditions d'un retour possible de l'horreur passée.

Bien que les lieux où a lieu le tourisme obscur soient toujours des espaces spécifiques renvoyant à des événements spécifiques, ces valeurs sont généralement considérées comme universelles. Les valeurs transmises et les formes prises pour les transmettre s'inscrivent dans la logique de la mondialisation, regardée comme un espace de communication universel (Lennon et Foley, $2000: 119$ ). Cela signifie à la fois que tout homme en tant qu'humain peut prendre le point de vue du tourisme obscur et que les valeurs transmises peuvent concerner tous les hommes se retrouvant dans une identité commune. Si une telle universalisation est partagée par le tourisme historique qui tend 
d'une manière générale à définir une identité humaine commune intégrant les différents ancrages nationaux ou locaux, le tourisme obscur a une spécificité : il ne s'ancre pas dans une contextualisation anthropologique des différentes cultures et communautés mises sur un pied d'égalité en tant que participant à la culture commune de l'humanité. L'universalité du tourisme obscur se fonde à un niveau presque ontologique et de toute manière infra-culturel : dans la réaction d'empathie de tout humain face à la douleur de ses semblables et dans l'identification des valeurs morales qui en sont tirées à un droit naturel à la vie plus qu'à une identité politique ou communautaire particulière.

C'est ce qui apparaît dans les deux manières dont cette universalisation est présentée comme se fondant sur la nature des différents événements ou par celle de la réaction devant eux. Williams $(2007: 135,146)$ précise bien ces deux orientations dans une même étude, alors que d'autres auteurs les analysent séparément. Du côté des événements, chaque massacre est considéré comme un cas particulier ou un exemple d'une catégorie générale, telle que massacre ou génocide. Cela se marque notamment par une internationalisation des formes mémorielles et muséales (Tunbridge et Ashworth, 1996 : 57-58). Du côté des visiteurs, tous les hommes sont considérés comme étant intéressés par ces événements, même s’ils ne sont pas a priori biographiquement concernés par un massacre précis (Tunbridge et Ashworth, 1996 : 122 ; Cole, 1999 : 102). La capacité de tout homme à ressentir souffrance et empathie ainsi que la dénonciation métaphysique du mal que l'homme peut faire à l'homme apparaissent comme transcendant les appartenances sociales, nationales ou communautaires. Dans les deux cas, les visiteurs sont considérés comme faisant l'expérience d'une identité commune. Une condition ou une identité humaine est fixée et dépasse celle de l'appartenance à des groupes sociaux avec des histoires et des identités particulières. Au-delà de l'identité historique et morale personnelle de chaque visiteur, le fait de partager cette histoire et ces valeurs avec les autres l'inscrit dans une communauté qui se veut universelle.

Ce sentiment d'universalité de l'identité des visiteurs est décrit par Williams comme pouvant se faire selon deux perspectives. D'un côté, comme c'est le cas en particulier des musées pour la paix, il s'agit d'affirmer des valeurs universelles sans les attacher ni à un événement ni à une communauté en particulier $(2007: 45,66)$. Cette volonté est marquée par la réunion dans le même site de références à de multiples événements et situations. C'est donc à partir de l'idée de valeurs communes à tous les humains que chaque situation particulière se révèle comme l'occasion d'en faire l'expérience. D'un autre côté, au contraire, ces valeurs peuvent être d'abord affirmées comme étant attachées à un événement particulier et à une communauté particulière reconnaissant dans ses valeurs propres quelque chose d'universel. Un tel processus est souvent décrit à propos du United States Holocaust Memorial Museum de Washington (Lennon et Foley, 2000 : 15 ; Linenthal, 2001 : 260-273). Williams se distingue du discours des institutions elles-mêmes et des travaux qui les reprennent ou les inspirent (Linenthal, 2001: XIIl).

Paul Williams est un des rares auteurs à émettre une réserve sur l'engagement civique associé au tourisme obscur (2007 : 105-107, 184, 190 ; voir aussi Sturken, 1997 : 17). Le fait de le présenter comme nécessaire s'accompagne d'une inquiétude quant à l'utilisation qui pourrait en être faite. Williams précise en effet que l'affirmation des valeurs ne doit pas se spectaculariser en perdant son contenu dans une forme de fierté nationaliste ou communautaire. Ce serait soit se réfugier derrière l'affirmation de valeurs pour les violer, soit s'en servir pour marquer sa propre supériorité par rapport à d'autres identifiés comme qui n'ont pas ces valeurs. Les valeurs d'un groupe social 
dominant peuvent être estimées comme universelles, comme ayant ainsi vocation à se propager jusqu'à être partagées par tous. Alors, si des États ou des identités nationales peuvent se fonder ou se confirmer sur une telle affirmation morale, leurs valeurs dépassent la dimension nationale pour s’inscrire dans une perspective universelle.

\section{Une pratique non culturelle de lieux culturels}

À côté des critiques centrées sur l'usage politique et social des musées et des sites historiques, celle qui est la plus partagée concerne la manière dont le point de vue patrimonial sur un événement se fait au détriment de son historicisation et de la transmission d'un savoir historien contextualisé. Cette situation apparait parfois comme corollaire de l'universalisation des valeurs : dans la mesure où les valeurs transmises sont universelles, il y a un désintérêt pour la spécificité historique de chaque événement qui est ainsi deshistoricisé (Walash, 1992 : 104-105, 130 ; Williams, 2007 : 105-107, 124, 137-139, 151). Le fait que la dimension morale donne son sens pédagogique au tourisme obscur signifie donc qu'il ne s'agit pas d'un tourisme culturel historique associé à un intérêt pour le savoir (Amirou, 2000). Il échapperait à ce que les théoriciens de l'éducation John Falk et Lynn Dierking nomment une "société d'apprentissage » (2000 : 211-214). Une telle exclusion s'inscrit dans une conception fermée de l'éducation considérée dans sa dimension d'apprentissage d'un savoir, contrairement à une conception plus ouverte, mettant l'accent sur l'acquisition de compétences qui ne sont pas seulement techniques mais également visent à juger d'un point de vue moral et civique (Löwenthal, 1998 : 121-122).

Cette dévalorisation de la transmission de valeurs prend deux formes en tant qu'elle s'oppose à la transmission d'un savoir historien. Ces valeurs ne sont pas des prédicats de l'événement historique et sont des constructions artificielles par opposition à une certaine conception du savoir historique qui transmet ce qui s'est véritablement passé. En même temps, elles font appel au ressenti des visiteurs et non à leur réflexion, ce qui peut avoir comme effet pervers un sentiment de plaisir associé à une trivialisation de l'événement.

Le seul élément de savoir transmis parfois évoqué est l'attestation de l'existence de l'événement grâce à des éléments matériels indiciels, sans pour autant historiciser ce qu'il a été. Lorsque la fonction de transmission d'un savoir historique est évoquée, c'est généralement pour regretter à la fois son absence et son remplacement par la transmission de valeurs. Cette critique est par exemple largement développée par Tim Cole (Huyssen, 1994, p 12 ; Cole, 1999, p. XVI). Le tourisme obscur est alors dénoncé comme la perte d'une mémoire vivante incarnée par les témoignages. Il serait un environnement historique naturel au profit d'un environnement artificiel, ainsi qu'un rapport corrompu et distordu au passé produisant un mirage en lieu et place de l'histoire. La rigueur de la connaissance historique et de sa transmission serait transformée par un pastiche ou un simulacre qui sont perçus comme la négation du projet historiographique. La réification du passé est déplorée à travers l'artificialisation de la mémoire. Les valeurs civiques et morales sont des significations données après l'événement, ou plus exactement données à sa mémoire. Elles n'apprennent rien sur son contexte politique, social, ou autre. C'est dans cette mesure que le tourisme patrimonial, qu'il s'agisse du tourisme historique en général ou du tourisme obscur en particulier, est perçu comme une spoliation de l'histoire, pour calquer le terme du 
géographe David Löwenthal (1998 ; voir aussi Lennon et Foley, 2000 : 163) ; au-delà du cas particulier des lieux du tourisme obscur, cette perspective est celle d'une certaine critique de la fonction même d'un rapport patrimonial à l'histoire. Reprenant explicitement les analyses de l'historien Pierre Nora (1997), l'archéologue Kevin Walash parle notamment de desinheritance (Walash, 1992 : 177).

Walash oppose cependant aux lieux de mémoire une transmission historienne, contrairement à Nora, ce qu'il nomme une mémoire vivante dont la forme est le témoignage. Il semble que pour lui le témoignage que Nora qualifie de mémoire naturelle soit associé à l'histoire comme deux manières de rendre compte de ce qui s'est passé. Or, Nora place l'histoire du côté du patrimoine dans la mesure où les deux sont des reconstructions présentistes du passé (Nora, 1997 : 35 ; Hartog, 2003 : 163-167). Il y a à ce niveau souvent un malentendu dans l'utilisation de la référence à Nora. Il vient de la manière dont la critique de l'artificialité des lieux de mémoire est reprise, sans prendre en considération la conception générale de la mémoire qui est celle de Nora. Ce dernier critique dans l'histoire et dans les lieux de mémoire leur artificialité qui en font des représentations présentistes. Ce n'est cependant pas ce qui est en jeu dans la critique du tourisme obscur qui se centre sur le regret d'une dimension d'apprentissage au profit d'une éducation des valeurs. L'artificialité n'est pas considérée comme étant l'effet de la méthode employée, mais de ce qui est visé ou de ce vers quoi fait signe en dernière instance le récit. Le récit historique n'est pas dit artificiel car il a sa fin en luimême. Ce qui est artificiel, c'est de s'en servir pour transmettre des valeurs dans une vision intéressée de l'histoire.

La valorisation du savoir historique par rapport à la transmission de valeurs a également une autre forme, attachée non seulement au contenu de ce qui est transmis, mais aussi à la manière de le transmettre : les valeurs sont acquises de manière sensible alors que l'histoire historienne s'adresse à l'activité réflexive. Le constat de cette différence est généralement en soi une dévaluation du ressenti associé au divertissement tenu pour vulgaire (Löwenthal, 1998 : 101-102). Cole pose par exemple la question de la pertinence de présenter au Musée-Mémorial d'Auschwitz-Birkenau et au United States Holocaust Memorial Museum des restes humains (1999 : 166-167). Il ne discute pas alors seulement de la question anthropologique du rapport aux restes humains et au respect des morts dans la culture occidentale, mais de l'incompatibilité de cette présentation avec la possibilité d'une expérience éducative. La morbidité suppose une relation affective et une esthétique du choc qui fait réagir les visiteurs en fonction de leur ressenti. C'est ce que montre d'une manière générale par exemple Williams en s'appuyant sur plusieurs autres exemples : les lieux du tourisme obscur présentent des ambiances destinées à provoquer un ressenti plus que des informations historiques ou des objets historicisés (2007 : 99 et suiv., 148). Il s'agit de scénographies pour faire sentir et faire réagir en provoquant une identification aux victimes et un sentiment d'incompréhension face à leurs souffrances, plus que de les comprendre. Les visiteurs ressentent une empathie et une horreur dont ils déduisent la nécessité d'affirmer des valeurs morales. Cole insiste sur ce point en évoquant de manière péjorative des pèlerinages pour qualifier ces visites (1999: 168).

Le tourisme obscur éloignerait alors de la compréhension et de la vérité de l'événement. Ce qui est ainsi transmis est largement considéré comme un spectacle où l'intérêt de l'événement historique se perdrait au profit d'un intérêt formel (Lennon et Foley, 2000 : 156). La critique de cette transmission effective échappant à la réflexion associée au savoir historien est indissociable de celle de la mise en place d'un spectacle à la place d'un dispositif d'apprentissage. Cette transmission est l'effet qu'Aristote reconnaît dans la tragédie et non dans la lecture d'un ouvrage d'histoire. La catharsis 
purge il est vrai les spectateurs par l'expérience de la pitié et de la terreur. S’il ne cite pas Aristote nommément, Anthony Seaton fait explicitement le rapprochement en utilisant une formule qui reprend presque mot pour mot celle de La Poétique (1996 : 240). Si le ressenti est dévalué par rapport à la réflexion, c'est en grande partie parce qu'il est associé non au savoir, mais au divertissement. La mise en avant du ressenti dans le tourisme obscur est généralement vue comme soit du voyeurisme, soit quelque chose de plaisant. Dans les deux cas, il s'agit bien de la perte d'un rapport authentique avec un événement associé à la mort. Il ne s'agit pas simplement de l'expérience vulgaire de quelque chose de sérieux, mais du risque que ce quelque chose soit luimême contaminé par le divertissement et perde son authenticité.

\section{Le risque d'une expérience récréative}

Tim Cole montre que la critique du voyeurisme va avec celle d'une trivialisation ou d'une kitschisation (1999 : 15, 73-93 ; également Sturken, 2007 ; Sharpley et Stone, 2009). La logique définie par le réalisateur Claude Lanzmann (Wadbled et Farache, $2013: 129$-132) dans ses fictions est alors utilisée. Cole reprend d'ailleurs une critique de la Liste de Schindler de Steven Spielberg directement inspirée des positions de Lanzmann lorsqu'il compare la trivialisation produite par le film à celle des lieux du tourisme obscur (Cole, 1999 : 73-93). La comparaison place ces lieux du côté de l'artificialité fictionnelle autant que fictive et lie explicitement cette question à celle de la trivialisation d'un spectacle perçu pour lui-même et non comme faisant signe vers un événement historique. Toute tentative de représentation tomberait dans ce travers, d'autant plus pour des génocides ayant laissé peu de traces authentiques et dont toute représentation serait une reconstitution. Aucune reconstitution d'ailleurs ne peut être à la hauteur de l'événement et est toujours inadéquate. Dans ce cas, non seulement la morbidité plaisante est-elle triviale, mais l'interprétation elle-même le devient dans la mesure où rendre compréhensible évite la confrontation avec l'horreur et l'incompréhensibilité de ce qui apparaît comme une autréité. Il y a soit une complaisance dans la représentation de l'horreur qui fait perdre de vue l'événement historique (Cole, 1999 : 114-115), soit une familiarisation avec elle qui fait perdre à l'événement son irréductibilité à toute compréhension (Lennon et Foley, 2000 : 157).

La conséquence est la crainte que ces événements soient associés à un sentiment de plaisir. Cela renvoie à la question aristotélicienne de la possibilité de prendre du plaisir à un spectacle qui suscite l'horreur. Au-delà de la catharsis qui requiert une attention au contenu, Aristote distingue en effet dans la tragédie ce qui produit le sentiment d'horreur et celui de plaisir : respectivement la forme de la représentation et le contenu représenté (Schaeffer, $2015:$ 165-170). C'est bien dans la mesure où l'intérêt se porte sur le spectacle et non plus sur l'événement auquel il fait référence qu'un sentiment de plaisir peut naître dans le tourisme obscur. Les lieux du tourisme obscur deviennent alors véritablement un parc d'attractions, puisque le plaisir n'est pas, comme dans un musée, destiné à encourager un apprentissage non formel, mais a sa valeur en luimême (Cole, 1999 : 110 ; Williams 2007 : 102 ; Sharpley et Stone 2009 : 13). De manière encore plus radicale que la comparaison avec un film de fiction qui demeure malgré tout une création à partir de l'histoire, la comparaison explicite avec Disneyland associe le divertissement à l'inauthenticité de quelque chose d'artificiel se présentant comme historique pour mieux produire un simulacre. La dimension de loisir deviendrait prépondérante et déterminerait en dernière instance les autres dimensions de l'expérience de visite ; l'événement pourrait se transformer en images à consommer. 
Le tourisme obscur serait alors une pratique récréative fondée sur le plaisir et non une pratique éducative.

Cette opposition correspond à celle proposée notamment par les géographes Yaniv Poria, Richard Butler, David Airey ou encore celle de Greg Richards, aussi géographe, pour distinguer le tourisme récréatif et une conception étendue du tourisme culturel incluant aussi bien sa dimension d'apprentissage de savoirs que celle d'éducation à des valeurs. Les deux approches complémentaires de ces auteurs permettent de définir de deux manières la différence affirmée dans les travaux sur le tourisme obscur. Les premiers proposent de réserver l'expression tourisme patrimonial (heritage tourism) aux visiteurs se sentant personnellement concernés d'une manière ou d'une autre par ce qu'ils visitent, contrairement au tourisme dans des lieux patrimoniaux (tourism in heritage places) désignant une visite de loisir désintéressée au sens où ce qu'ils visitent n'est pas vécu comme étant leur patrimoine (Poria et al., 2003 : 248). Selon une logique kantienne, ce désintéressement associe explicitement cette seconde pratique à un jugement de goût plaisant, à l'opposé d'une connaissance ou d'un jugement moral (Kant, 1993 : 70-83). De son côté, Richards oppose les expériences touristiques créatives associées à une prise de conscience ou à un apprentissage où le visiteur construit son savoir ou son identité aux pratiques récréatives associées au plaisir qui serait passif au sens où il ne participerait pas au développement du visiteur (2001: 64). Cette double distinction renvoie à la dévaluation du plaisir par rapport à des activités jugées plus sérieuses et moins futiles. Elle s'inscrit dans une logique platonicienne selon laquelle les représentations artificielles éloignent de la vérité de ce qui est représenté. Le point de vue patrimonial impliquerait un rapport à des simulacres éloignant de la vérité de l'événement historique qui ne pourrait être transmise qu'en s'adressant à la réflexion et non au ressenti (Platon, 1966 : 600c-605d ; 1993 : 234b236b) : il s'agit d'une réification qui réduit la compréhension historique au lieu de la générer. Le tourisme induisant un tel rapport à un événement historique ne peut donc lui-même être qualifié d'historique.

Malgré ces critiques, il semble que ce ne soit pas le ressenti et le plaisir qui soient en eux-mêmes condamnés. Ce qui est dénoncé, c'est le risque qu'ils deviennent la raison d'être du tourisme obscur. Dans certain cas, l'un comme l'autre apparaissent en effet comme étant tolérés, dans la mesure où ils sont instrumentalisés pour transmettre un savoir historien sur les événements. La critique de la trivialisation et du plaisir dans une société de loisir ne signifie donc pas nécessairement un refus de ces dimensions.

Si le ressenti est dévalorisé par rapport à la réflexion, il peut apparaître comme y menant. Dans la perspective d'une reconnaissance d'un caractère sensible de l'apprentissage non formel (Dudley, 2010 ; Meunier, $2011: 33-34$ ), le ressenti serait susceptible de donner un savoir plutôt que des valeurs. Edward Linenthal (2001 : 212216) et Paul Williams (2007 : 39 et suiv.) vont notamment en ce sens lorsqu'ils évoquent la situation des restes humains d'une manière qui s'oppose à celle de Tim Cole (1999). Ils considèrent que la dimension sensible n'implique pas nécessairement une trivialisation marquée par la dépersonnalisation et la déshistoricisation qui peuvent être une expression de l'horreur de ce qui s'est passé et de la désindividualisation de la mort de masse. Pour eux, ces restes sont des témoins de ce qui s'est passé au même titre que les autres objets, et attestent donc aussi de l'existence des victimes. En faire l'expérience rapproche - et n'éloigne donc pas - de la compréhension de l'événement.

De la même manière, le plaisir et le loisir peuvent être des moyens ou des stratégies pour capter et soutenir l'attention et l'intérêt des visiteurs (Lennon et Foley, 2000 : 157). Cela s'inscrit dans une conception du plaisir partagée aussi bien par la muséologie 
de l'éducation que par la géographie du tourisme culturel : ce plaisir peut être accepté à condition d'être au service d'un dynamique d'apprentissage qualifiée d' " edutainment » (Tunbridge et Ashworth, 1996 : 266 ; Hooper-Grennhill, 2007 : 33). Sans doute en raison du contenu des ressentis associés à des émotions négatives, la possibilité de prendre du plaisir lors de l'apprentissage de savoirs ou de valeurs (Alderson et Payne, 1987 : 23-24) n'est par contre jamais mentionnée dans le cas particulier du tourisme obscur.

C'est à ce niveau que la problématique aristotélicienne de la catharsis ne se pose pas : dans les rares cas où elle est tolérée, la possibilité d'un plaisir reste associée à la forme touristique et non au contenu spécifique du tourisme obscur. C'est la confusion entre les deux qui semble critiquée. Il s'agit d'une situation où la mise en avant du ressenti en vient à se tourner contre sa fonction initiale : elle n'est même plus capable de transmettre des valeurs civiques et morales puisque le contenu de ce qui est représenté est devenu indifférent. Non seulement la transmission du contenu de ces valeurs pose un problème dans une " société d'apprentissage » dans la mesure où elles ne disent rien de l'événement historique, mais la forme de cette transmission contient en elle les conditions de la perte même de ce contenu. Il se produit alors une confusion qui se reflète dans la notion de tourisme obscur, comme l'ont remarqué notamment William Miles (2002 : 1175-1178) et Philip Stone (2006 : 69, 145-160). Dans une société de consommation et de loisir, on en vient à ne plus prendre garde au contenu du spectacle consommé pour donner une valeur à cette consommation et à l'expérience récréative elles-mêmes, de sorte que des choses qui auraient dû être séparées sont mises sur un plan analogue : des lieux de loisir et des lieux historiques lointains dans le temps ou dans l'espace sont inclus dans le tourisme obscur au même titre que des lieux de massacres contemporains qui concernent encore leurs visiteurs. Pour marquer la différence, Miles propose de parler de « patrimoine le plus obscur » (darkest heritage) ou de " tourisme le plus obscur» (darkest tourism) pour désigner les formes de tourisme dont l'expérience ne devrait pas devenir triviale et plaisante.

\section{Conclusion. Considérer ce que vivent effectivement les touristes}

Définir ce qui caractérise le tourisme obscur de manière fonctionnaliste nécessite une subsomption des cas particuliers dans un cadre général où les caractéristiques spécifiques aussi bien des sites visés que des individus les visant sont considérées comme négligeables. Or, plusieurs auteurs s'inscrivant dans cette perspective générale estiment que l'universalité proclamée des valeurs n'est en fait pas réalisée et cache une profonde disparité en fonction de la spécificité tant des sites que de leurs visiteurs. Il y a en effet des dissonances radicales et irréductibles dans tout patrimoine. Le même site peut renvoyer à des expériences entièrement différentes (Tunbridge et Ashworth, 1996 ; Williams, $2007: 151,153,171$ ). La question est celle d'une conscience universelle au-delà des situations culturelles locales. Si par exemple Williams en conclut la nécessité de complexifier et de différencier la pratique du tourisme obscur en fonction des différentes situations, il ne remet pas fondamentalement en question la perspective fonctionnaliste. Il la complexifie en introduisant la différenciation du tourisme obscur en sous-catégories.

Il serait plutôt possible d'y voir l'indice de la nécessité de changer de paradigme, en partant non de fonctions générales définies demandant à être reprises 
contextuellement, mais plutôt des contextes physiques et personnels pour voir la manière dont ils entrent effectivement en relation afin de déterminer de manière immanente le mode de perception qui est actualisé. Ce serait définir le tourisme obscur en tant que pratique, à partir de la manière dont il est effectivement vécu dans des situations particulières. La perspective s'inverse : la question de l'étude des fonctions sociales et culturelles devient dépendante des expériences subjectives particulières.

Il s'agit de considérer avec Maurice Halbwachs (1997) qu'un mode de perception ne se réduit pas à une signification dans l'économie générale d'une société et d'une culture. Pour ce sociologue, les rôles sociaux et culturels n'existent que dans la mesure où ils sont investis par les individus qui les prennent et à chaque fois les déplacent. Les lieux et les visiteurs ne sont pas des acteurs dont l'action remplit une fonction ou joue un rôle prédéfini, quelles que soient leurs histoires. Ils sont des agents qui créent à chaque fois une relation particulière à ce qu'ils perçoivent, de sorte qu'une modalité de perception n'est pas un cadre dans lequel ils s'inscrivent mais le résultat immanent de leur activité. Les modes de perception ne sont pas des réalités préexistantes que les individus prendraient de manière passive, mais plutôt la résultante ou l'intégration des situations particulières où des positions fonctionnelles sont prises. Inversant la logique fonctionnaliste, la compréhension d'une expérience est alors indissociable de la manière dont elle est vécue par ceux qui l'ont réalisée, en partant du principe que comprendre une action sociale revient à la saisir à des fins interprétatives et à des significations subjectives. Il faut donc renverser l'approche fonctionnaliste qui domine la bibliographie proposant une caractérisation générale du tourisme obscur, pour lui préférer des études de cas. La définition du tourisme obscur serait alors une généralisation analytique produite de manière immanente par des individus percevant un site (Yin, 2014 : 40-49) et non par des règles de fonctionnement social les subsumant.

\section{Bibliography}

Alderson, William et Shirley Payne, 1987, Interpretation of Historic Sites, Nashville, AASLH [American Association for State and Local History] Press.

Amirou, Rachid, 2000, Imaginaire du tourisme culturel, Paris, Presses universitaires de France.

Bloom, Thomas, 2000, « Morbid Tourism: A Postmodern Market Niche with an Example from Althorpe », Norwegian Journal of Geography, no. 54, p. 29-36.

DOI : $10.1080 / 002919500423564$

Cole, Tim, 1999, Selling the Holocaust, From Auschwitz to Schindler, Londres-New York, Routledge.

Dean, Carolyn, 2003, "Empathy, Pornography, and Suffering ", Differences, vol. 14, no 1, p. 88-124.

DOI : 10.1215/10407391-14-1-88

de Certeau, Michel, 1975, L'Écriture de l'histoire, Paris, Gallimard.

Deleuze, Gilles, 1989, "Qu'est-ce qu'un dispositif ? », dans Michel Foucault philosophe. Rencontre internationale de Paris, 9, 10, 11 janvier 1988, Paris, Seuil, p. 185-195.

Dudley, Sandra, 2010, " Museum Materialities. Objects, Sense and Feeling ", dans Sandra Dudley (dir.), Museum Materialities. Objects, Engagements, Interpretations, Londres, Routledge, p. 1-17.

Falk, John et Lynn Dierking, 2000, Learning from Museums. Visitor Experiences and the Making of Meaning, Walnut Creek, Altamira-Press.

Foley, Malcom et John Lennon, 1996, "JFK and Dark Tourism: A Fascination with Assassination », International Journal of Heritage Studies, no. 2, p. 198-211. 
DOI : $10.1080 / 13527259608722175$

Halbwachs, Maurice, 1997, La Mémoire collective, Paris, Albin Michel.

Hartog, François, 2003, Régimes d'historicité, Paris, Seuil.

Hooper-Greenhill, Eilean, 2007, Museum and Education, New York, Routledge.

Huyssen, Andreas, 1994, « Monument in the Post-modern Age », dans James Young (dir.), The Art of Memory: Holocaust Memorial in History, Munich, Prestel Verlag, p. 9-17.

Huyssen, Andreas, 1995, Twilight Memories: Making Time in a Culture of Amnesia, New York, Routledge.

Huyssen, Andreas, 2000, " Present Past: Media, Politics, Amnesia », Public Culture, vol. 12, no 1 , p. 21-38.

DOI : 10.1215/08992363-12-1-21

Huyssen, Andreas, 2011, La hantise de l'oubli. Essais sur les résurgences du passé, Paris, Kimé.

Kant, Emmanuel, 1993, Critique de la faculté de juger, Paris, Vrin.

Ledoux, Sébastien, 2012, « Écrire une histoire du 'devoir de mémoire' », Le Débat, no 170, p. $175-185$.

DOI : 10.3917/deba.170.0175

Lennon, John et Malcolm Foley, 2000, Dark Tourism. The Attraction of Death and Disaster, Londres, New York, Continuum.

Linenthal, Edward, 2001, Preserving Memory: The Struggle to Create America's Holocaust Museum, New York, Columbia University Press.

Löwenthal, David, 1985, The Past Is a Foreign Country, New York, Cambridge University Press.

Löwenthal, David, 1998, Possessed by the Past. The Heritage Crusade and the Spoils of History, Cambridge, Cambridge University Press.

Malinowski, Bronislaw, 1970, Une théorie scientifique de la culture, Paris, Point Seuil.

Meunier, Annick, 2011, Le potentiel éducatif de l'exposition. La mise en scène des objets ethnographiques : l'analyse de l'influence éducative de différentes mises en exposition, Sarrebruck, Éditions universitaires européennes.

Miles, William, 2002, « Auschwitz: Museum Interpretation and Darker Tourisme », Annals of Tourism Research, vol. 29, no 4, p. 1175-1178.

DOI : 10.1016/So160-7383(02)00054-3

Misztal, Barbara, 2004, « The Sacralization of Memory », European Journal of Social Theory, vol 7 , no 1, p. 67-84.

DOI : $10.1177 / 1368431004040020$

Nora, Pierre, 1997, " Entre mémoire et histoire. La problématique des lieux », dans Pierre Nora (dir.), Les lieux de mémoire, tome 1 : La République, Paris, Gallimard, coll. « Quarto », p. XVII-XLII.

Novick, Peter, 1999, The Holocaust in American Life, Boston, Houghton Mifflin.

Phillips, Robert, 1995, « Contesting the Past, Constructing the Future. History, Identity and Politics in School ", dans John Arnold, Kate Davies et Simon Ditchfield (dir.), History Heritage: Consuming the Past into Contemporary Culture?, Shaftsbury (RU), Donhead, p. 237- 248 .

DOI : $10.1111 / 1467-8527.00069$

Platon, 1993, Le Sophiste, Paris, Garnier Flammarion.

Platon, 1966, La République, Paris, Garnier Flammarion.

Poria, Yaniv, Richard Butler et David Airet, 2003, " The Core of Heritage Tourism », Annals of Tourism Research, vol. 30, no 1, p. 238-254.

DOI : 10.1016/So160-7383(02)00064-6

Richards, Greg, 2001, Cultural Attractions and European Tourism, New York, CABI Publishing.

DOI : 10.1079/9780851994406.0000

Schaeffer, Jean-Marie, 2015, L'Expérience esthétique, Paris, Gallimard. 
Seaton, Anthony V., 1996, " Guided by the Dark: From Thanatopsis to Thanatourism », International Journal of Heritage Studies, no 2, p. 234-244.

Seaton, Anthony V., 2009, " Purposeful Otherness: Approaches to the Management Thanatourism », dans Richard Sharpley et Philip Stone (dir.), The Darker Side of Travel. The Theory and Practice of Dark Tourism, Bristol-Buffalo-Toronto, Channel View Publications, p. 75-108.

Sharpley, Richard, 2005, "Travels to the Edge of Darkness: Towards a Typology of Dark Tourism ", dans Christ Ryan, Stephen J. Page et Michelle Aicken, Taking Tourism to the Limits: Issues, Concepts and Managerial Perspectives, Amsterdam, Elsevier, p. 215-226.

DOI : 10.1016/B978-0-08-044644-8.50023-0

Sharpley, Richard et Philip Stone, 2009, "(Re)-Presenting the Macabre: Interpretation, Kitschification and Authenticity », dans Richard Sharpley et Philip Stone (dir.), The Darker Side of Travel. The Theory and Practice of Dark Tourism, Bristol-Buffalo-Toronto, Channel View Publications, p. 109-128.

Stone, Philip, 2006, « A Dark Tourism Spectrum: Toward a Typology of Death and Macabre Related Tourist Sites, Attraction and Exhibition ", Tourism: an Interdisciplinary, International Journal, vol. 54, no 2, p. 145-160.

Stone, Philip, 2009, " Dark Tourism: Morality and New Moral Spaces », dans Richard Sharpley et Philip Stone (dir.), The Darker Side of Travel. The Theory and Practice of Dark Tourism, Bristol-Buffalo-Toronto, Channel View Publications, p 56-72.

Sturken, Marita, 2007, Memory, Kitsch, and Consumerism from Oklahoma City to Ground Zero, Durham, Duke University Press Books.

Tunbridge, John et Gregory Ashworth, 1996, Dissonant Heritage: The Management of the Past as a Resource in Conflict, Hoboken (NJ), John Wiley and Sons.

Wadbled, Nathanaël et Michèle Farache, 2013, « Le différent de la représentation : poétiques de l'expérience concentrationnaire ", dans Alina Crihana (dir.), Communication interculturelle et littérature, no 19 , Mémoire, littérature et identité, vol. 1 Les récits de vie : mémoire, histoire et fictions identitaires, Galati, Roumanie, Institutul European, p. 127-143.

Walash, Kevin, 1992, The Representation of the Past. Museums and Heritage in the Postmodern World, Londres-New York, Routledge.

Wallace, Mike, 1989, " Mickey Mouse History: Portraying the Past at Disney World », dans Leon Warren et Roy Rosenzweig (dir.), History Museums in the United States, Chicago, University of Illinois Press, p. 158-179.

DOI : 10.1215/01636545-1985-32-33

Williams, Paul, 2007, Memorial Museums: The Global Rush To Commemorate Atrocities, Oxford-New York, Berg.

Yin, Robert, 2014, Case Study Research. Design and Methods, Thousand Oaks (CA), Sage.

\section{Notes}

1 D'autres termes ont été proposés pour caractériser l'intérêt touristique pour la mort et la souffrance s'orientant vers d'autres types d'expérience : thanatourisme (Seaton, 1996), tourisme morbide (Bloom, 2000), tourisme douloureux (Sharpley, 2005).

2 Cette différence entre apprentissage et éducation ne recouvre pas exactement celle entre learning et education utilisée dans la muséologie anglo-saxonne essentiellement pour opposer un apprentissage actif à un apprentissage passif (Hooper-Greenhill, 2007 : 1-5). Il s'agit plutôt d'opposer d'un côté la transmission d'un savoir destiné à être appris intellectuellement ou réflexivement et d'un autre côté la transmission de valeurs ou de manières d'être, d'agir, de penser, etc.

\section{References}

Electronic reference

Nathanaël WADBLED, « Les fonctions du tourisme obscur », Téoros [Online], 35, 1| 2016, 
Online since 05 September 2016, connection on 07 November 2017. URL :

http://teoros.revues.org/2851

\section{About the author}

\section{Nathanaël WADBLED}

Doctorant, Sciences de l'information et de la communication, Université de Lorraine / Muséologie, médiation, patrimoine, Université du Québec à Montréal ; nathanael_3009@yahoo.fr

\section{Copyright}

\section{(2) $\odot \Theta \Theta$}

La revue Téoros est mise à disposition selon les termes de la Licence Creative Commons Attribution - Pas d'Utilisation Commerciale - Pas de Modification 4.0 International. 